\title{
Internal charge behaviour of nanocomposites
}

\author{
J Keith Nelson $^{1}$ and John C Fothergill ${ }^{2}$ \\ ${ }^{1}$ Department of Electrical, Computer and Systems Engineering, Rensselaer Polytechnic \\ Institute, Troy, NY 12180, USA \\ ${ }^{2}$ Department of Engineering, University of Leicester, Leicester LE1 7RH, UK
}

Received 23 December 2003

Published 1 March 2004

Online at stacks.iop.org/Nano/15/586 (DOI: 10.1088/0957-4484/15/5/032)

\begin{abstract}
The incorporation of $23 \mathrm{~nm}$ titanium dioxide nanoparticles into an epoxy matrix to form a nanocomposite structure is described. It is shown that the use of nanometric particles results in a substantial change in the behaviour of the composite, which can be traced to the mitigation of internal charge when a comparison is made with conventional $\mathrm{TiO}_{2}$ fillers. A variety of diagnostic techniques (including dielectric spectroscopy, electroluminescence, thermally stimulated current and photoluminescence) have been used to augment pulsed electro-acoustic space charge measurement to provide a basis for understanding the underlying physics of the phenomenon. It would appear that, when the size of the inclusions becomes small enough, they act cooperatively with the host structure and cease to exhibit interfacial properties, leading to Maxwell-Wagner polarization. It is postulated that the particles are surrounded by high charge concentrations in the Gouy-Chapman-Stern layer. Since nanoparticles have very high specific areas, these regions allow limited charge percolation through nano-filled dielectrics.

The practical consequences of this have also been explored in terms of the electric strength exhibited. It would appear that there was a window in which real advantages accrue from the nano-formulated material. An optimum loading of about $10 \%$ (by weight) is indicated.
\end{abstract}

(Some figures in this article are in colour only in the electronic version)

\section{Introduction and background}

Rather surprisingly, the current push to develop nanomaterials based on nanotechnology has not focused much on the opportunities for dielectric materials, but rather centred on optical and mechanical applications, driven by the microelectronics industry. Nonetheless, the few examples in the literature provide encouragement that this is likely to be fertile ground. Furthermore there are good theoretical reasons why the pursuit of nanomaterials for dielectric applications may have particular promise. Some of these have been reviewed by Lewis [1] and Fréchette [2]. While the technology of 'nanodielectrics' is in its infancy, one may speculate that it will be possible to self-assemble nanodielectrics by providing chemical structures with 'hooks' that provide preferential attachment points for the nanostructured materials, allowing automatic and predictable self-assembly.
In general, the use of conventional fillers does little for the electric strength of polymeric materials; indeed it usually impacts negatively. However, there are other important reasons for using fillers, including improving discharge resistance, improving thermal or electrical conductivity, matching thermal expansion and controlling costs. In this context, it is thought that fundamental to controlling the dielectric strength of insulating polymers is the cohesive energy [3] density and the associated free volume [4] of a polymer structure. This may be gauged by examining the changes in electric strength (up to a factor of 10) exhibited by most polymers as they are taken through their glass transition temperature [5]. In the simplest situation, the bonding of a polymer to filler can be expected to give a layer of 'immobilized' polymer. The size of this layer is critical to the global properties (electrical, mechanical and thermal) of the composite and it is this interaction zone that is key to determining the behaviour. It is also clear that, from the 
dielectric standpoint, the filler material may give rise to spacecharge accumulation and the associated Maxwell-Wagner polarization due to the implanted interfaces. Furthermore, the macroscopic theories of interfacial polarization do not incorporate a molecular approach since the response is given by relaxation equations if the wavelength is large in comparison with molecular dimensions. In considering pre-breakdown high-field conduction in pure materials, the existence of localized states within the energy bandgap (close to the conduction or valence bands) is usually invoked, so giving rise to a mobility edge for electron (or hole) transport [6]. These states are essentially localized on individual molecules. This is because, unlike the strong covalent bonds of elemental crystalline solids, the intermolecular binding arises from weak van der Waals' forces that do not allow inter-molecular electronic exchange. In this paper we show that many of these observations appear to be challenged when the particulate inclusions are of nanometric dimensions.

\section{Sample formulation}

The material used in this investigation was a bisphenolA epoxy (Vantico CY1300 + HY956) with $23 \mathrm{~nm}$ and $1.5 \mu \mathrm{m}$ (average diameter) $\mathrm{TiO}_{2}$ particles incorporated in the host matrix (to form nano- and micro-composite materials respectively). The resin was selected because it was benign (i.e. without other fillers or dilutents), had a low initial viscosity and had a glass transition below $100^{\circ} \mathrm{C}$. Transmission electron microscopy indicated that the nanoparticulates ranged in size from 10 to $100 \mathrm{~nm}$ with $70 \%$ smaller than $30 \mathrm{~nm}$ in diameter.

The formulation and processing of the planar specimens (having thicknesses between 500 and $750 \mu \mathrm{m}$ ) have been described previously [7]. Because of their small size, surface interactions for nanoparticles, such as hydrogen bonding, become magnified. This means that the particles tend to agglomerate and dispersion in resins is quite difficult, even though such polymers should be relatively compatible. Hence, in the case of nanoparticles, large shear forces are needed in the mixing process to obviate unwanted clustering of the particles. Field emission SEM would indicate that the nanoparticles are well dispersed, although some agglomeration was present, yielding a few clusters up to $500 \mathrm{~nm}$, despite the precautions taken. However, it is surmised that a sufficient amount of the nanoparticles were dispersed to permit the composite to exhibit properties characteristic of the nanofilled material. For many electrical characterizations, the cast film was provided with evaporated $100 \mathrm{~nm}$ aluminium electrodes.

Recessed breakdown specimens (exhibiting a quasiuniform field) were prepared by the use of a mould having multiple polished spherical protrusions of $6.35 \mathrm{~mm}$ diameter so as to form a plurality of recessed specimens [4] in one operation (see figure 1(a)). Divergent field specimens were created by moulding around electrolytically etched tungsten needles having a well-characterized tip radius of about $4 \mu \mathrm{m}$ shown in the photomicrograph of figure 1(b). In all cases, careful optical microscopic examination of the specimens was undertaken to reject those with agglomerations, cavities and other defects. (a)

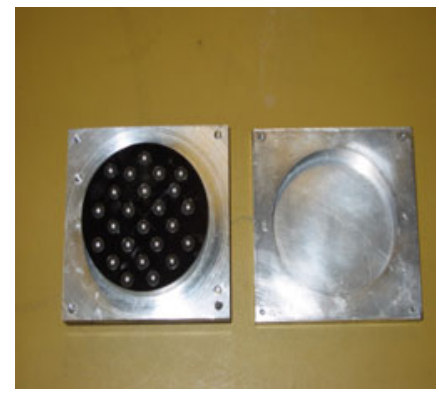

(b)

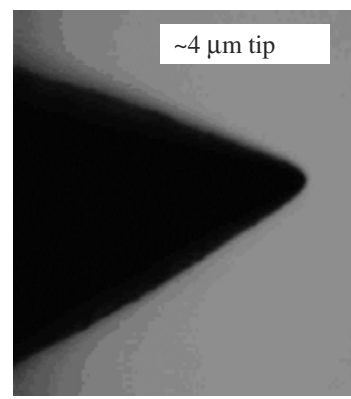

Figure 1. (a) Mould for multiple recessed specimens

(b) Electrolytically etched needle.

Table 1. Photoluminescence data for resin samples.

\begin{tabular}{|c|c|c|c|c|c|c|}
\hline \multirow[b]{2}{*}{$\begin{array}{l}\text { Ex. } \lambda \\
(\mathrm{nm})\end{array}$} & \multicolumn{2}{|c|}{ Base resin } & \multicolumn{2}{|c|}{$10 \%$ micro } & \multicolumn{2}{|c|}{$10 \%$ nano } \\
\hline & $\begin{array}{l}\mathrm{Pk} \\
\lambda\end{array}$ & $\begin{array}{l}\mathrm{Pk} \\
\mathrm{Mag}\end{array}$ & $\begin{array}{l}\mathrm{Pk} \\
\lambda\end{array}$ & $\begin{array}{l}\mathrm{Pk} \\
\text { Mag }\end{array}$ & $\begin{array}{l}\mathrm{Pk} \\
\lambda\end{array}$ & $\begin{array}{l}\mathrm{Pk} \\
\mathrm{Mag}\end{array}$ \\
\hline 280 & 413 & 7.0 & 411 & 25.5 & 418 & 29.6 \\
\hline 320 & 409 & 21.5 & 411 & 35.8 & 420 & 44.6 \\
\hline 340 & 407 & 65.4 & 405 & 128.1 & 412 & 35.2 \\
\hline 360 & 408 & 85.8 & 406 & 151.2 & 423 & 14.1 \\
\hline
\end{tabular}

\section{The effect of particulate size on the composite environment}

\subsection{Photoluminescence}

Laminar moulded specimens using both micro- and nano-particulates were subjected to photoluminescence measurements as depicted in table 1 for excitation wavelengths ('Ex. $\lambda$ ' in the table) from 280 to $360 \mathrm{~nm}$. The shift in the peak wavelength in the presence of the nanoparticles (sixth column in table 1 , ' $10 \%$ nano, $\mathrm{Pk} \lambda$ ') implies that the emitting species have had their environment altered. On the assumption that the emission is excimeric in origin, this suggests that the nanoparticles may cause minor conformational changes sufficient to bind the excimer units more tightly. The magnitude of the peak emission in the nanocomposite case is also behaving in an entirely different way (decreasing with increasing excitation wavelength) when compared with the response of the conventional micron-sized filler.

\subsection{Dielectric spectroscopy}

Some insight into the way that the incorporation of materials of nanometric dimensions affects the dielectric properties may be obtained by examining the variation of the real and imaginary components of relative permittivity as a function of temperature and frequency. This has been done for the $\mathrm{TiO}_{2}$ filled material at temperatures of 293, 318, 343, 368 and $393 \mathrm{~K}$ using a Solartron HF frequency response analyser (type 1255) in combination with a Solatron Dielectric Interface, type 1296. Generally, at least two samples were tested in order to check reproducibility. The response at different temperatures was similar but shifted along the frequency axis, suggesting that the various processes are thermally activated.

Figure 2 shows relative permittivity Bode plots obtained for the base resin and the $10 \% \mathrm{w} / \mathrm{w}$ micro- and nanocomposites at $393 \mathrm{~K}$; figure 3 shows the loss tangent $\left(\varepsilon^{\prime \prime} / \varepsilon^{\prime}\right)$ 

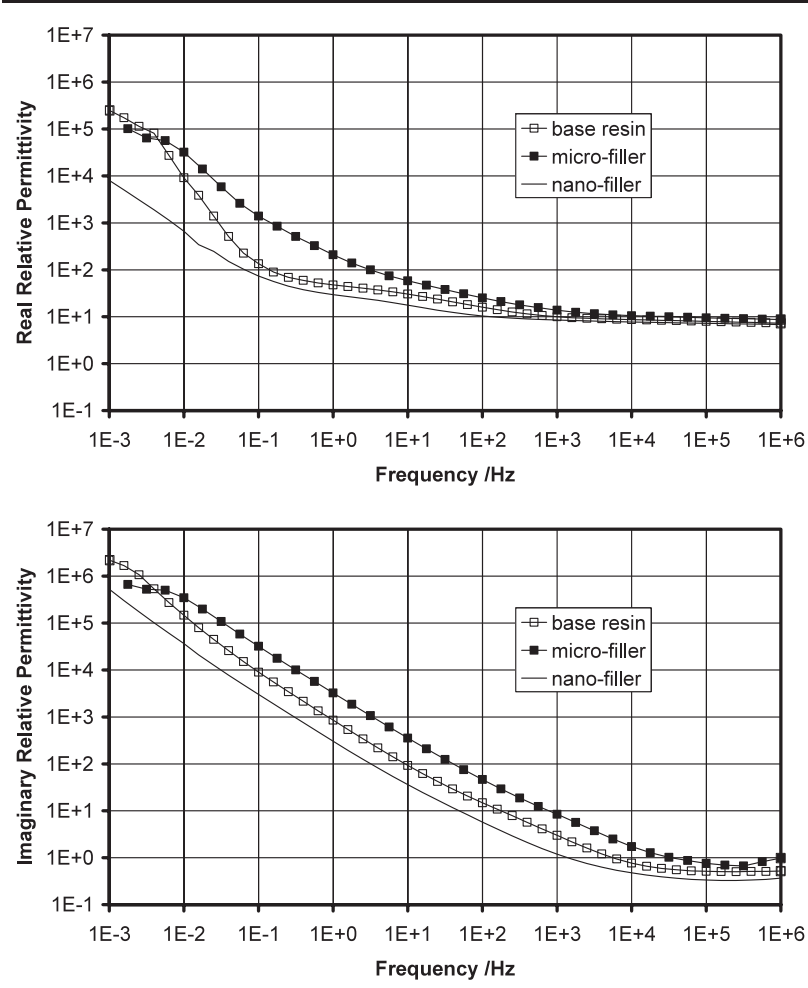

Figure 2. Real and imaginary relative permittivity of base resin, micro- and nano-composite materials at $393 \mathrm{~K}$.

calculated from these data. At higher frequencies, the micronfilled material has a higher real relative permittivity; this is presumably due to the high relative permittivity of the filler $(\approx 99)$. It is instructive to compare the real part of the relative permittivity of the different materials at (for example) $1 \mathrm{kHz}$ where the low frequency effects do not dominate. At $393 \mathrm{~K}$, the base resin exhibits a real relative permittivity of 9.99 . Computations of the composite permittivity, $\varepsilon_{\mathrm{C}}$, for the 'conventional' (micron-sized) $\mathrm{TiO}_{2}$ filler based on the Lichtenecker-Rother logarithmic law of mixing applicable to chaotic or statistical mixtures:

$$
\log \varepsilon_{\mathrm{C}}=y_{1} \log \varepsilon_{1}+y_{2} \log \varepsilon_{2}
$$

(where $y_{1}, y_{2}$ are the volume fractions of the two components having relative permittivities $\varepsilon_{1}, \varepsilon_{2}$, respectively) yield a value of 10.1. This compares with the measured value of 13.8 under the same circumstances, perhaps indicating some interfacial effects. However, in marked contrast, the nanocomposite at $1 \mathrm{kHz}, 393 \mathrm{~K}$ exhibits a measured value of 8.49 , which is significantly less than that of the base resin matrix. This is true at all the temperatures measured. This surprising result, which has been confirmed using a second test utilizing a different spectrometer, suggests that the interaction zone surrounding the nanoparticles is having a profound effect on the dielectric behaviour of the nanocomposite. The nanoparticles appear to restrict end-chain or side-chain movement of the epoxy molecules. This is consistent with a change in epoxy morphology, the 'anchoring' of end-chains on the nanoparticles, or, possibly, a change in composition perhaps associated with an increased proportion of hardener close to the surface of the nanoparticles.

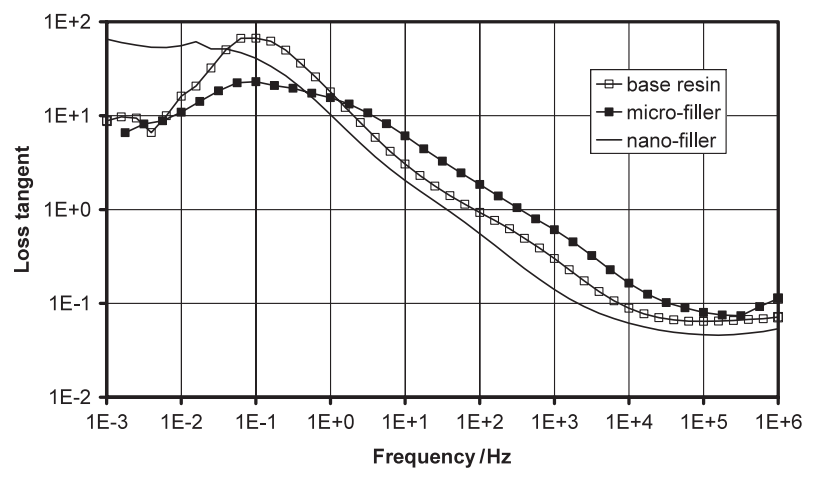

Figure 3. Loss tangent of base resin, micro- and nano-composite materials at $393 \mathrm{~K}$

In the frequency range $0.1-100 \mathrm{~Hz}$, the base-resin and nanocomposite behave in a similar way. There is possibly a small dielectric relaxation in this range, consistent with the findings of Griseri [15], shown by the real permittivity increasing with decreasing frequency. For the micro-filled particles this has an activation energy of $1.7 \mathrm{eV}$ whereas, for the nano-filled material, this drops to $1.4 \mathrm{eV}$. The smaller response and activation energy associated with the nanofilled material again suggest that the nanoparticles restrict chain movement. The microcomposite real permittivity, however, shows a marked increase with decreasing frequency. This clearly must be associated with the microparticles and is possibly a Maxwell-Wagner interfacial polarization. Although the frequency for such a polarization mechanism may be considered rather high, the interfaces introduced by the particles are, on average, quite close together (of the order of $1.5 \mu \mathrm{m}$ ) and so the time constant for the build-up of charge at these interfaces would be much less than that usually associated with interfacial polarization. The base resin exhibits classic Maxwell-Wagner interfacial polarization below a frequency of $0.1 \mathrm{~Hz}$ with slopes of -2 and -1 on the real and imaginary permittivity Bode plots [8] and a peak in the loss tangent at $0.1 \mathrm{~Hz}$, as shown in figure 3 . The microcomposite also displays this peak in loss tangent, which therefore must be due to interfacial polarization at the electrodes. At very low frequencies, the dielectric behaviour of the base resin and microcomposite become very similar as electrode effects dominate. The thickness of the samples was approximately $750 \mu \mathrm{m}$, approximately 500 times the intermicroparticle distance. It is interesting that the deviation between real permittivities of the microcomposite and the base-resin starts to occur at approximately 500 times the frequency of $0.1 \mathrm{~Hz}$, giving some support for the theory that the microcomposite was exhibiting Maxwell-Wagner polarization due to the microparticle interfaces.

The nanocomposite shows a quite different behaviour at low frequencies, below $0.1 \mathrm{~Hz}$. Instead of the real part of the relative permittivity having a slope of -2 , it is parallel to the imaginary part with a slope of -1 . This is indicative of what Jonscher [8] termed a 'low-frequency dispersion (LFD)' or what Dissado and Hill [9] refer to as 'quasi-DC (QDC)' behaviour. The flattening out of the $\tan \delta$ response at lower frequencies is also symptomatic of QDC behaviour. This behaviour is observed when charge carriers have some limited freedom of movement within a material. Typically carriers, 
(a) $10 \% 1.5 \mu \mathrm{m} \mathrm{TiO}{ }_{2}$ particles, $3 \mathrm{kV}$ after 1 hour

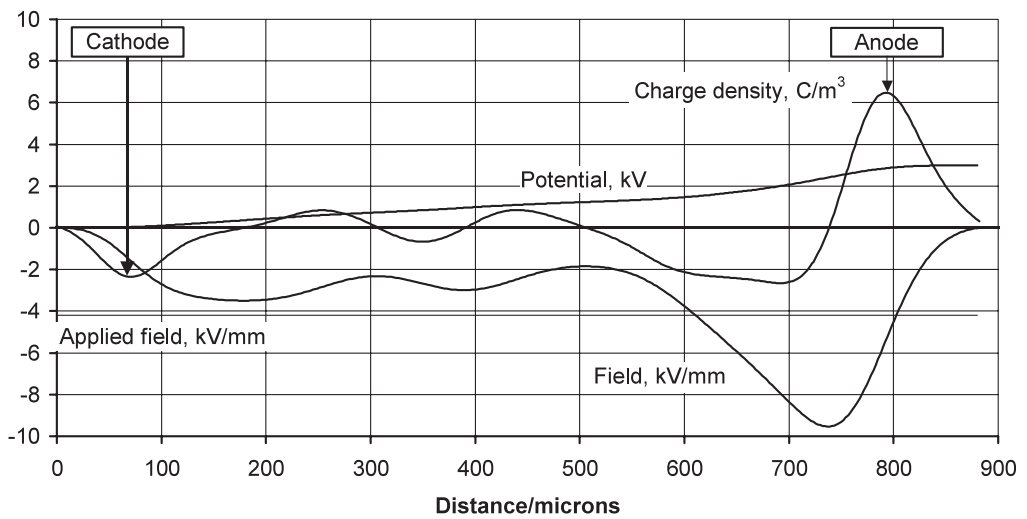

(b)

$10 \% 38 \mathrm{~nm} \mathrm{TiO}{ }_{2}$ particles, $3 \mathrm{kV}$ after 1 hour

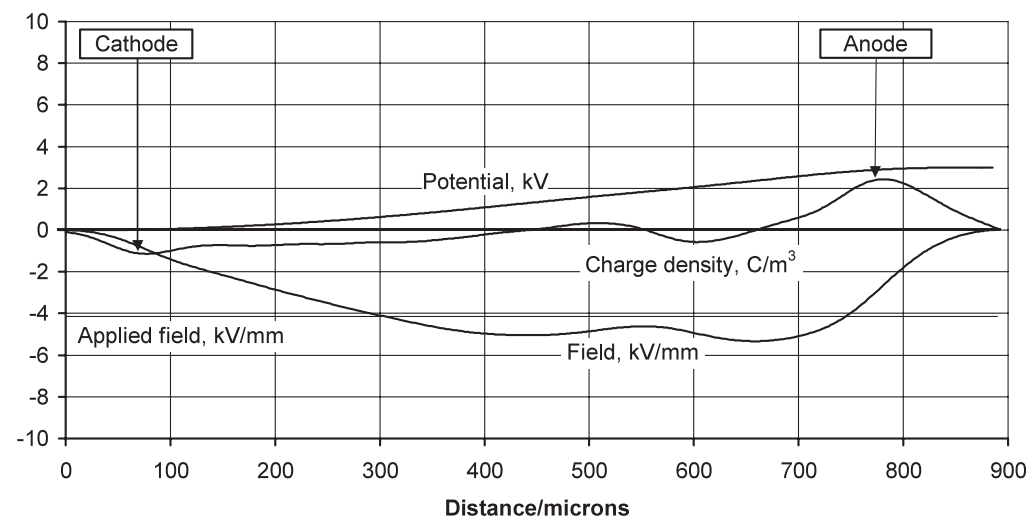

Figure 4. Typical PEA space charge measurements: (a) micro-filled material, (b) nano-filled material.

under the influence of the electric field, follow tortuous paths that would not allow transport completely through the material. As well as the nanoparticles causing morphological changes to the epoxy during crosslinking, there would be a 'dielectric interaction' layer surrounding such particles. Lewis has considered the dielectric effects on the material surrounding nanoparticles [1] and, in particular, the production of a Stern layer on the surface of the particles surrounded by a diffuse Gouy-Chapman layer [10]. Such a layer has been considered for solids [11] and good agreement between theory and results has been demonstrated. This Gouy-Chapman-Stern layer is highly conductive in comparison with the matrix, and charge movement through it would be relatively facile. It is difficult to calculate the thickness of this layer in a dielectric since this has only been considered for liquid electrolytes and for solid crystals containing defects. If one considers the latter case and, as a first approximation, assumes defects every $10 \mathrm{~nm}$ (i.e. about the length of a chain of an epoxy molecule) a defect concentration of $10^{24} \mathrm{~m}^{-3}$ would be implied. The screening length would then be approximately $3 \mathrm{~nm}$ with enhanced conductivity extending for several times this length [12]. If such layers overlapped between several nearby nanoparticles, charge movement over limited distances would be facilitated. Since the distances between nanoparticles would be reasonably random, the path lengths of such carriers would form a distribution, possibly akin to a Poisson distribution. Dissado and Hill modelled this field-enhanced percolation as a fractal circuit and showed that a QDC response would be shown in
Table 2. Time constant estimates for charge (PEA) and light (electroluminescence) decay in $\mathrm{TiO}_{2}$.

\begin{tabular}{lcl}
\hline & $\begin{array}{c}38 \mathrm{~nm} \\
\mathrm{TiO}_{2}\end{array}$ & $\begin{array}{l}1.5 \mu \mathrm{m} \\
\mathrm{TiO}_{2}\end{array}$ \\
\hline Charge decay (s) & 22 & 1800 \\
Light decay (s) & $<60$ & 1200 \\
\hline
\end{tabular}

the dielectric relaxation spectrum. We can therefore conclude that the movement of charge in this material is enhanced by the presence of the nanoparticles due to the high surface area/volume ratio and this would be expected to greatly change the dielectric behaviour of such materials. This is consistent with the marked change in time constant discussed in section 8 and typified in table 2 .

Reduction of the particulate loading from 10 to $1 \%$ (by weight) did not have any very obvious fundamental changes, but the nano-filled material then does start to exhibit a low frequency response more typical of the base resin and microfilled material, suggesting that changes engineered by the nanomaterials do require loadings greater than a few per cent.

\section{The impact on internal charge}

In an earlier contribution, a pulsed electro-acoustic (PEA) study was conducted to assess the field distortions in the bulk. The method has been described elsewhere, e.g. [13]. 
(a) $10 \% 1.5 \mu \mathrm{m} \mathrm{TiO}{ }_{2}$ particles, $3 \mathrm{kV}$

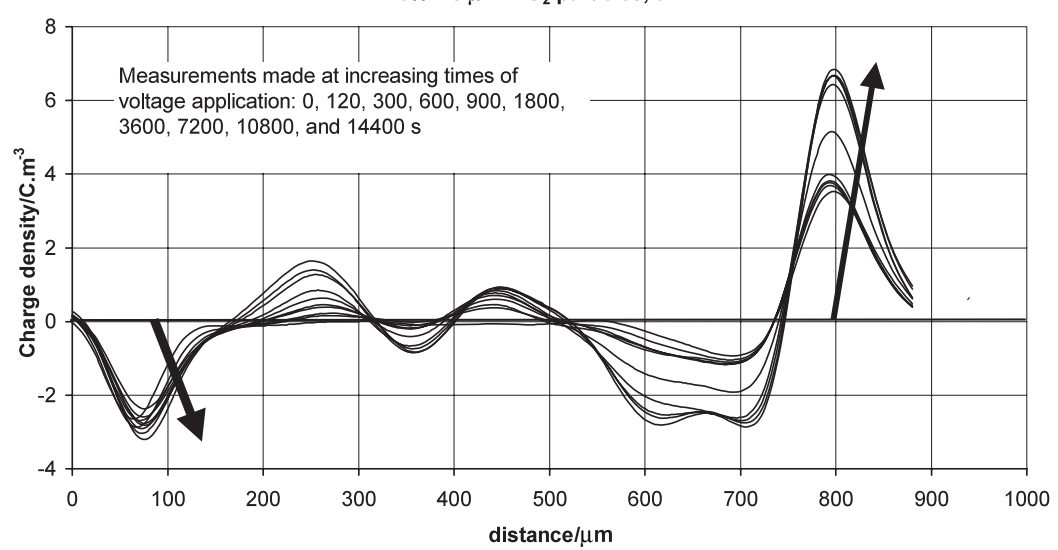

(b)

$10 \% 38 \mathrm{~nm} \mathrm{\textrm {TiO } _ { 2 }}$ particles, $3 \mathrm{kV}$

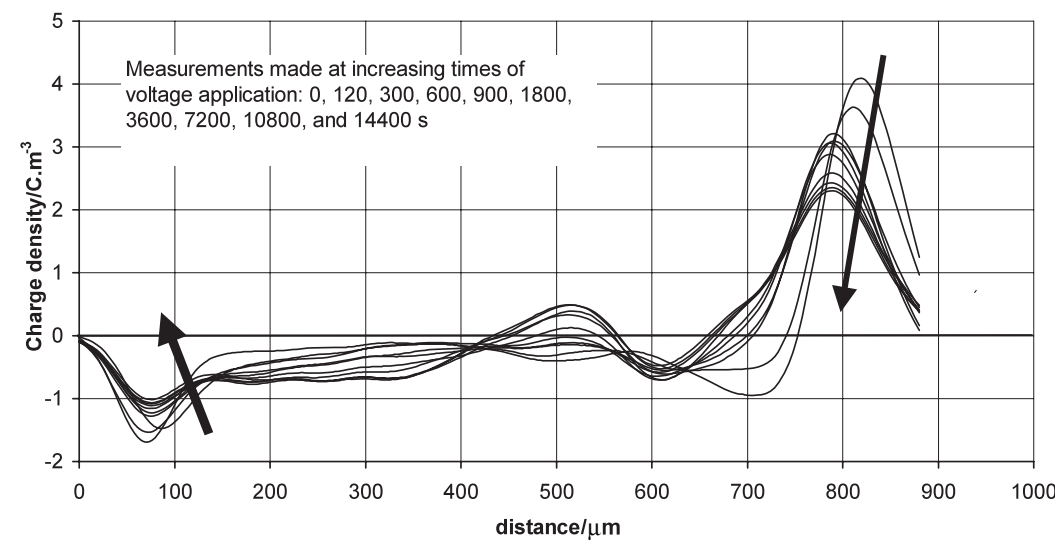

Figure 5. (a) Charge dynamics in micro-filled material. The arrows show the charge development with time (2 min to 4 h). (b) Charge dynamics in nano-filled material.

Laminar samples were subjected to direct voltages and the initial distribution of stress shows little deviation from the nominal $4.2 \mathrm{kV} \mathrm{mm}^{-1}$ uniform level across the bulk.

Figures 4(a) and (b) show typical PEA measurements on micro- and nano-filled materials after $1 \mathrm{~h}$ of stressing at $3 \mathrm{kV}$ DC. The curves show the charge density, the electric field and potential in a plane in the sample at a given distance from the sensing electrode (the cathode in this case). It can be seen that the charge levels in the nano-filled materials are considerably reduced in comparison to the microcomposite. The curves have been calculated by filtering with a fifthorder Gaussian filter, deconvolving with a space-charge-free (PMMA) reference and calibrating. The calibration included accounting for the exponential attenuation of the signal through the sample; epoxy is quite lossy anyway but, when filled, scattering of the acoustic wave occurs which further attenuates the signal. The microcomposite shows strange variations in space charge which, as shown later, are reasonably stable in terms of their position. Similar effects have been reported by Bambery and Fleming [14]. Griseri [15] has attributed this to a stable interaction between the field caused by the space charge and the consequently induced polarization. This has been shown to persist for long times (> weeks).

Figures 5(a) and (b) show how the charge distribution changes with time for the micro- and nano-filled materials. Of particular note are the large heterocharge accumulations near the electrodes for the microcomposites. These cause large image charges on the electrodes which are increasing with time and resulting in increasingly large field enhancements liable to initiate breakdown. The converse is true for the nano-filled materials in which the charges may move more easily. The electrode charges decrease with time in this case. Figures 6(a) and (b) show the corresponding changes of local electric field intensity with time for the two systems. The microcomposite field is dominated by the heterocharge at the anode and is increasing and considerably higher than the average (applied) field of $4.2 \mathrm{kV} \mathrm{mm}^{-1}$. The field in the nanocomposite appears to be 'evening out', i.e. it is becoming closer to the average field with time throughout the material.

In order to demonstrate the difference between these two systems, figure 7 shows the maximum field intensity (i.e. at the point in the sample where the field is highest) as a function of time for the two systems. The maximum field in the nanocomposite is reasonably constant and perhaps reducing with time. According to this analysis (which is limited by the resolution of the PEA system), the field in this case is never more than approximately $125 \%$ of the applied field. The field in the microcomposite, however, increases rapidly as charge is injected and unable to move away from the electrode. The field in this case is approximately $235 \%$ of the applied field. Transient PEA studies, such as these, permit the establishment and decay of charge profiles to be viewed in time. These show that there are very substantial differences in the time constants associated with the migration and decay of charge for 

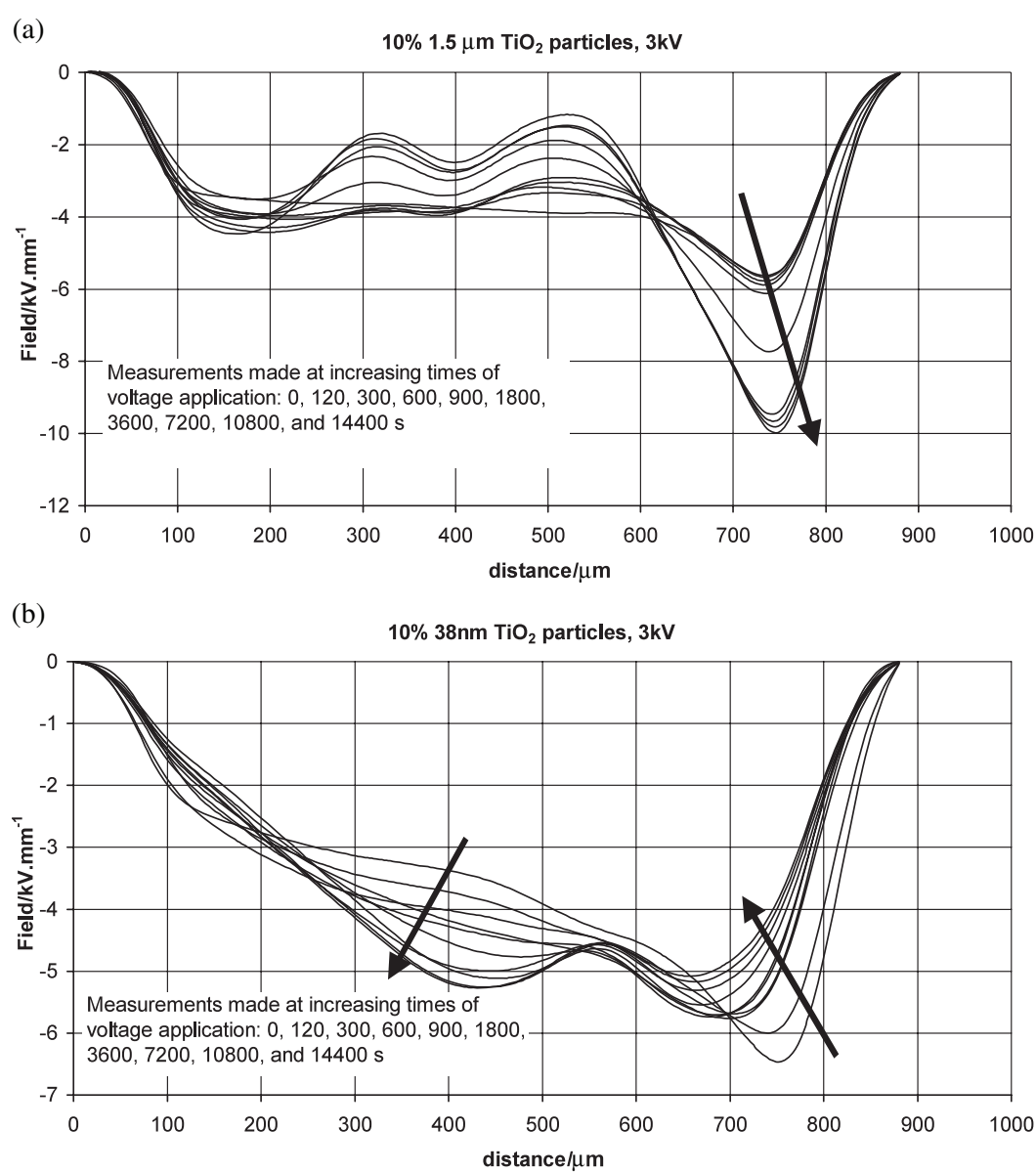

Figure 6. Development of the electric field in (a) micro-filled material and (b) nano-filled material.

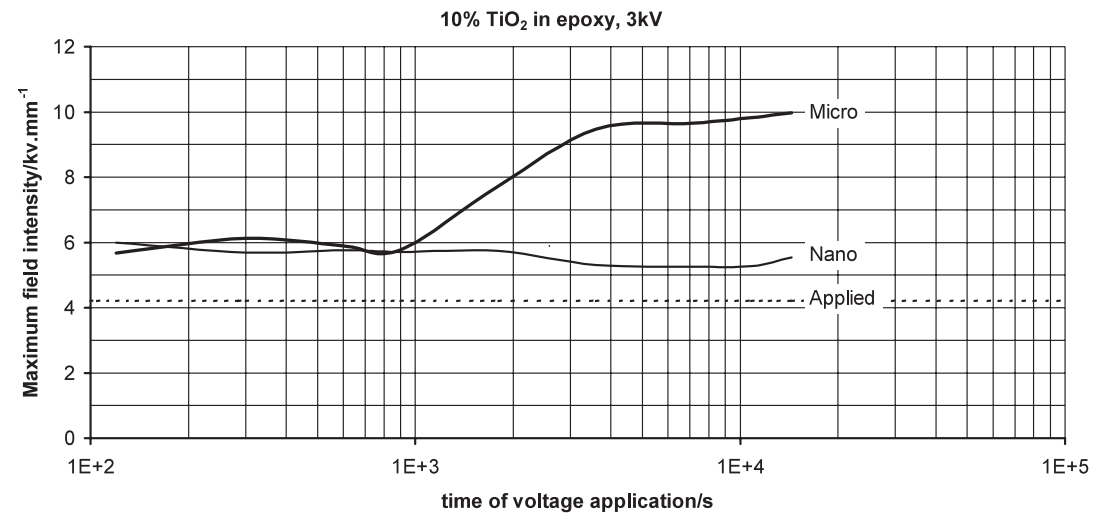

Figure 7. The maximum field intensity with the two systems as a function of time, compared to the average 'applied' field.

the micro- and nano-composites, as is illustrated later in table 2 in comparison with optical electroluminescence emission. In contrast to the microcomposite, the decay of charge in the nano-filled $\mathrm{TiO}_{2}$ is very rapid, with insignificant homocharge remaining after just $2 \mathrm{~min}$. Although there is some injection of negative charge at the cathode, the nano-filled material is characterized by much less charge accumulation.

\section{Electroluminescence studies}

The light emission from a $\sim 4 \mu \mathrm{m}$ point moulded into the resin samples is depicted in figure 8 for a $10 \%$ loading. The pre- discharge electroluminescence is measured with a 13-dynode EMI 9789B photomultiplier tube having a bialkali spectral response connected in scintillation counting mode (i.e. the light is determined by counting pulses during a fixed interval, usually $60 \mathrm{~s}$ ). Two hours was allowed for the photocathode to stabilize before measurements were attempted. The field, $E$, in figure 8 is that calculated at the individual tip based on [16]:

$$
E=\frac{2 V}{r \ln (4 d / r)}
$$




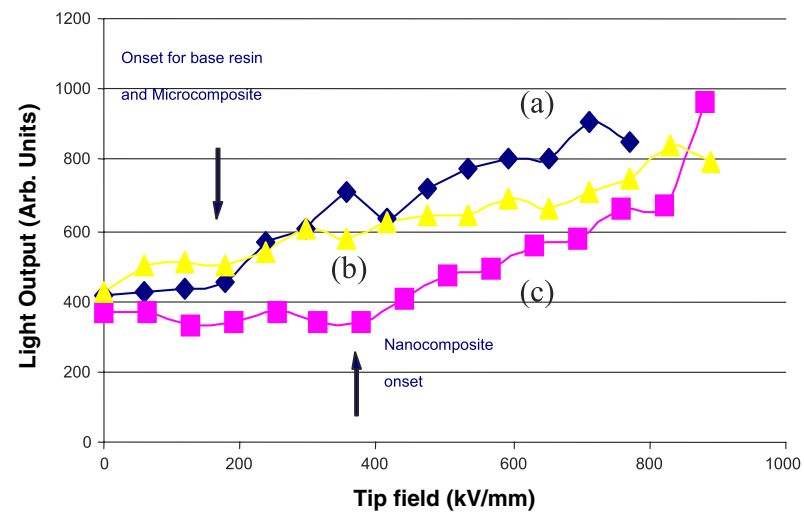

Figure 8. Electroluminescence characteristics in $\mathrm{TiO}_{2}$ composites. (a) Base resin, (b) $10 \%$ micro (c) $10 \%$ nano.

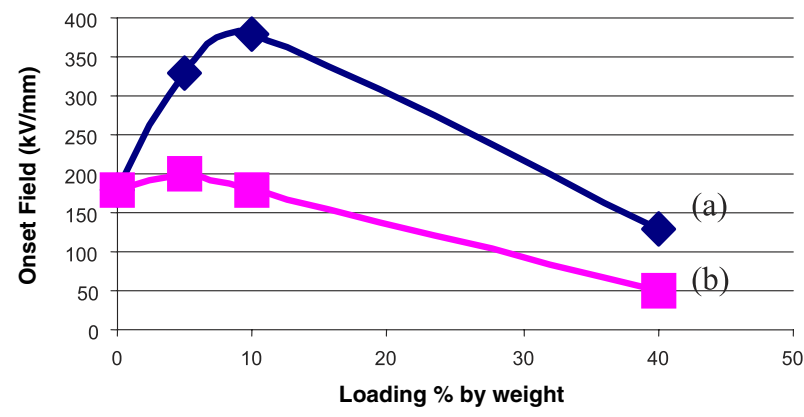

Figure 9. Electroluminescence onset field as a function of $\mathrm{TiO}_{2}$ loading. (a) $23 \mathrm{~nm}$, (b) $1.5 \mu \mathrm{m}$.

where $r$ is the tip radius and $d$ is the inter-electrode gap. This may over-estimate the field since there is likely to be considerable homo-charge accumulation around such injecting points. However, in this equation, the calculated field is proportional to the applied voltage and so the argument below would still be internally consistent.

While the level of activity for the nanomaterial is generally somewhat less, the salient feature is the light onset level. The nanomaterial requires $400 \mathrm{kV} \mathrm{mm}^{-1}$ to register output above the background count whereas both the base resin and the micromaterial start emitting at stresses which are only half that value-about $180 \mathrm{kV} \mathrm{mm}^{-1}$. This compares with the $178 \mathrm{kV} \mathrm{mm}^{-1}$ found by Griseri [17] using uniform fields in a similar resin system. However, this comparison may be fortuitous since the previous study speculated that the emission is the result of a bipolar charge recombination mechanism. In this divergent field case, it is more likely that the light results from the downward transition of excited species formed by electron injection in the high tip field. When the electroluminescence output is examined as a function of loading (figure 9), it is clear that enhancement in the onset is again a maximum at about $10 \%$, as is indicated later for the electric strength.

Electroluminescence measurements have also been made as a function of time to observe the way in which the materials react to a step change in stress of $600 \mathrm{kV} \mathrm{mm}^{-1}$. Figures 10(a) and (b) depict the dynamics of light emission for $10 \%$ nano- and micro-composites. (The time response of the base resin is of the same form as shown in (a) for the nanocomposite.) Comparison of these under both switchon and switch-off transients indicate that the two materials
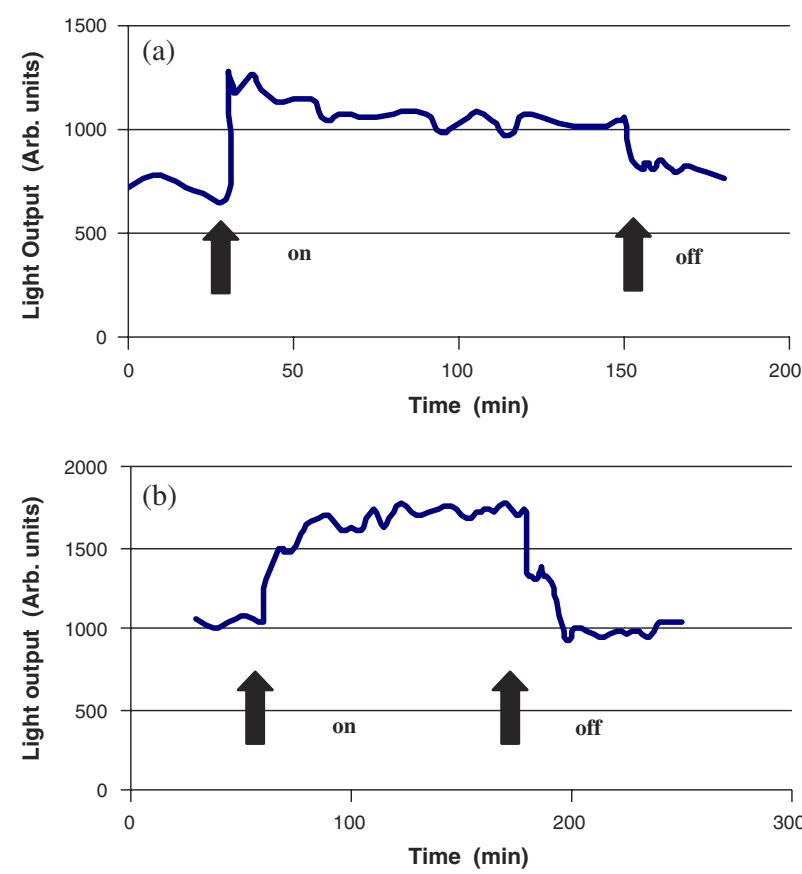

Figure 10. Dynamics of electroluminescence in response to step changes in electric field. (a) $23 \mathrm{~nm} \mathrm{TiO}_{2}$, (b) $1.5 \mu \mathrm{m} \mathrm{TiO}$.

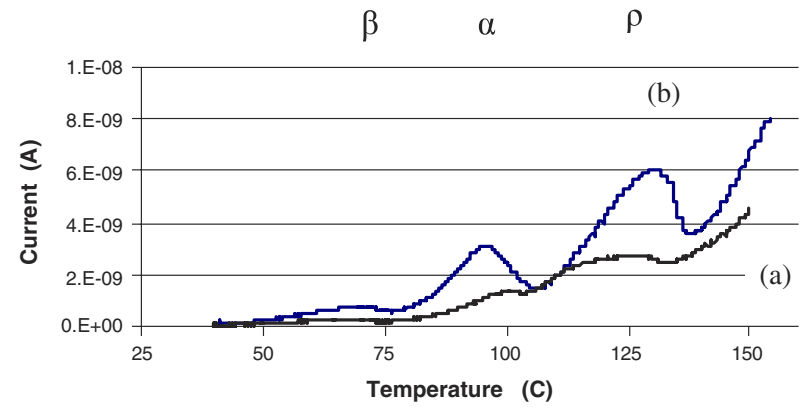

Figure 11. Thermally stimulated current spectra for (a) $10 \% 23 \mathrm{~nm}$ $\mathrm{TiO}_{2}$ and (b) $10 \% 1.5 \mu \mathrm{m} \mathrm{TiO}$ fillers.

respond very differently, as will be discussed at greater length later. However, it is also important to recognize that light is emitted for a period after the applied field is removed, strongly suggesting that it is the Poisson (i.e. space charge) field and not the Laplacian field that is intimately involved with electroluminescence.

\section{Thermally stimulated currents}

Laminar samples of both micro- and nano-filled resin were subjected to thermally stimulated discharge having been poled at $115^{\circ} \mathrm{C}$ at a stress of $5.5 \mathrm{kV} \mathrm{mm}{ }^{-1}$. The temperature ramp rate was $0.05^{\circ} \mathrm{C} \mathrm{s}^{-1}$. Typical plots for the two different types of material are shown in figure 11 , curves (a) and (b).

The glass transition temperature, $T_{\mathrm{g}}$, for the base resin is $89^{\circ} \mathrm{C}$, and differential scanning calorimetry measurements [7] have already demonstrated that $T_{\mathrm{g}}$ can be expected to change slightly with the $\mathrm{TiO}_{2}$ filler size for this resin. Accordingly, the TSC peaks at about $90^{\circ} \mathrm{C}$ may be associated with the main chain relaxation (the $\alpha$ peak). Similarly, the peak at about $70{ }^{\circ} \mathrm{C}$ can be associated with the $\beta$ relaxation. However, the 


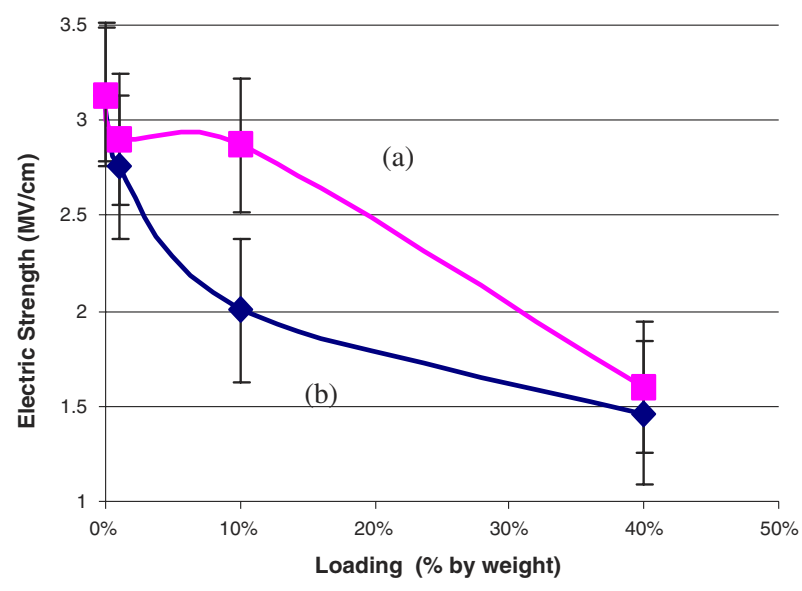

Figure 12. Electric strength of epoxy/ $\mathrm{TiO}_{2}$ composites. (a) $23 \mathrm{~nm}$, (b) $1.5 \mu \mathrm{m}$.

characteristics above $100{ }^{\circ} \mathrm{C}$ are very different indeed for the two filler sizes. This is the region identified as due to the release of space charge in epoxy resins [18]— the $\rho$ peak shown in figure 11 .

\section{Impact on electric strength}

Short-term electric strength measurements have been measured under DC conditions with a ramp rate of $500 \mathrm{~V} \mathrm{~s}^{-1}$. Figure 12 depicts the mean breakdown gradient (for a population of 10 samples) for the base resin, as well as the micro- and nano-composites as a function of filler loading (\% by weight). The advantage in electric strength attributable to the nano-sized filler is clear and an optimum loading of about $10 \%$ is indicated. For high loadings (close to the percolation limit), however, the advantages are eroded and the degradation in mechanical properties makes such very high loadings unattractive.

\section{Appraisal}

The large interaction zone in nano-filled polymers with reduced mobility (free volume [19]) should be accompanied by a significant change in electrical properties. Studies of electrical behaviour thus provide an opportunity both for a fundamental study of this interaction zone and also an opportunity for optimizing performance for specific and critical applications.

The finding that conventional fillers are accompanied by substantial bulk charge accumulation is clearly a factor in the common experience of the lower electric strengths exhibited for filled materials. The mitigating effects of nanoparticles provide encouragement that nanocomposites can be engineered with strengths that are commensurate with the base polymer. The dielectric strength advantages of nano-filled epoxy indicated here (figure 12) are in line with other cited cases in which the addition of nanometric materials have shown substantial benefits, particularly with regard to improvements in voltage endurance [20,21]. Although enhancements above the strength of the base resin have been seen, there is clearly a window of opportunity in being able to enhance other attributes of an insulating structure (thermal conductivity, mechanical properties, discharge resistance, etc) without the usual penalty of sacrificing electric strength. Not only does the incorporation of nanoparticles yield a dielectric strength close to that of the base polymer, but figure 13 also demonstrates that the $\beta$ parameter (dispersion) is unchanged by the addition, in contrast to the micro-filled material where a significant change of slope in the Weibull plot is indicated.

While the presumed reduction of free volume on the substitution of nanoparticles may be instrumental in improving the electric strength [22], the results presented here also strongly suggest that the improvements in electric strength may be linked to the control of the internal charge within the bulk. The electroluminescence onset studies reported here suggest that the large surface area inherent to nanoparticles has created a mechanism for electron scattering which will skew the energy distribution with beneficial results, i.e. a higher voltage is required for light onset. However, in seeking reasons for the marked differences seen in many aspects of behaviour when nanoparticulates are incorporated, figure 10 would seem to be pivotal. The escalation of light in microcomposites over a period of about an hour following energization suggests that the tip field is augmented by the establishment of heterocharge (positive) in front of the point cathode. Indeed, the emission of light following the removal of the applied stress dictates that the tip field is sustained by charge in the bulk. Careful examination of previous PEA results [7] indicates that such a region of charge is, indeed, formed when the infilled material is of large $(\mu \mathrm{m})$ dimension. In contrast, the nanomaterial exhibits the maximum electroluminescence on switch-on, indicating that any charge that is accumulated acts to shield the point electrode and reduce the high-field light emission. This effect will also be incorporated in figure 8 since there was sufficient time allowed for charge modification to take place. Although not shown in figure 8 , cases were documented where the onset of light occurred measurably earlier for the microcomposite than for the base resin.

The PEA method also allows the decay of charge to be estimated following the removal of the applied field. Table 2 provides estimates of the decay time constants obtained from the decay of the electrode image charges in a PEA experiment for $\mathrm{TiO}_{2}$ nano- and micro-composites in comparison with electroluminescence decay. While the absolute numbers are not comparable because of the differing geometries, nevertheless, the very substantial differences brought about by the filler size are demonstrated by both techniques and, again, point to the effects of internal fields.

However, it remains to identify the nature and origin of the charge that gives rise to this behaviour. As suggested earlier, interfacial polarization is a prime candidate and is now strongly suggested by the characteristics of figure 4(a). Charges trapped at the interfaces formed by the microparticles will be neutralized by charges of opposite sign conveyed to the interfaces by Ohmic conduction giving rise to a TSC transient. This means that the nature of the TSC peak (and even its polarity) will depend on both the relative permittivity and the conductivity of the constituent materials. Following the work of van Turnhout [23], the TSC transient due to the annihilation of charge, $\sigma$, is given by

$$
i(t)=\frac{\mathrm{d} \sigma}{\mathrm{d} t} \frac{\left[\varepsilon / \varepsilon_{1}-g(T) / g_{1}(T)\right]}{\left[s / s_{1}+g(T) / g_{1}(T)\right]\left(1+\varepsilon s_{1} / e_{1} s\right)}
$$

where the ratios of the permittivities $\varepsilon / \varepsilon_{1}$ and the conductivities $g / g_{1}$ will determine the polarity of the discharge current during 


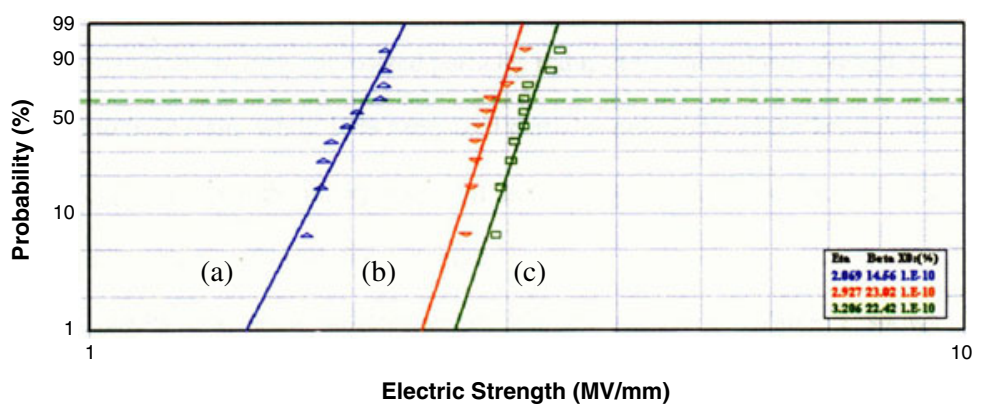

Figure 13. Composite breakdown statistics plotted as a Weibull distribution. (a) Micro, (b) nano, (c) base resin.

the TSC thermal ramp. Consequently, for micro-filled $\mathrm{TiO}_{2}$, a negative Maxwell-Wagner peak is sometimes experienced, particularly at low poling temperatures. However, the poling temperature used in figure $11\left(115^{\circ} \mathrm{C}\right)$ is above $T_{\mathrm{g}}$ and thus the $\rho$ peak should be fully developed [23] and the position of the peak is independent of the poling conditions as has been found in this study. The significant finding here is that the nanocomposite does not exhibit the marked $\rho$ peak characteristic of Maxwell-Wagner interfacial effects in the conventional material.

The PEA results taken in conjunction with the dielectric spectroscopy studies suggest that significant interfacial polarization is implied for conventional fillers, which is mitigated in the case of particulates of nanometric size, where a short-range highly immobilized layer develops near the surface of the nanofiller $(\sim 3 \mathrm{~nm})$. This bound layer, however, influences a much larger region surrounding the particle in which conformational behaviour and chain kinetics are significantly altered. This interaction zone is responsible for the material property modifications, especially as the curvature of the particles approaches the chain conformation length of the polymer. Evidence suggests that the local chain conformation and configuration play major roles in determining the interactions of a polymer with nanofillers [19]. Recent work [24] on the visco-elastic behaviour of nanocomposites has indicated that particulates of nanometric dimensions contributed to a process of tethered entanglement that might reflect in the environmental change indicated in table 1 . The tethering density increases with nanomaterials because of the increased anchoring associated with enhanced particle surface area. This is consistent with the upward shift in the temperature of the $\alpha$ peak depicted in figure 11 and could be expected to be associated with a beneficial reduction in free volume.

In the case of nanofillers, there is evidence that a grafted layer is formed by the absorption of endfunctionalized polymers onto the surface especially when the functional groups are distributed uniformly along the polymer backbone. Hence the local chain conformation is critical in determining the way in which bonding takes place, and thus the cohesive energy density, which is known to be an important factor in establishing the 'intrinsic' dielectric strength [3]. The defective nature of nanoscale particles can be expected to enhance the bonding if chemical coupling agents (CVD coatings on nanoparticles or triblock copolymers) are employed. Such methods would seem to be indicated as an obvious next step in the commercial exploitation of nanodielectrics.

\section{Acknowledgments}

The authors are indebted to the UK EPSRC under whose auspices some of this work was undertaken, to the University of Leicester for the provision of facilities, and to Dr Teyssèdre of the Universite Paul Sabatier, France, who undertook the photoluminescence measurements. Contributions made by Ms $\mathrm{Y} \mathrm{Hu}$ and $\mathrm{Mr} \mathrm{J}$ Thiticharoenpong are also gratefully acknowledged.

\section{References}

[1] Lewis T J 1994 Nanometric dielectrics IEEE Trans. Dielectr. Electr. Insul. $1812-25$

[2] Frechette M F et al 2001 Introductory remarks on nanodielectrics Annual Report of the Conf. on Electrical Insulation and Dielectric Phenomena (Piscataway, $\mathrm{NJ}$ : IEEE) pp 92-9

[3] Sabuni H and Nelson J K 1976 Factors determining the electric strength of polymeric dielectrics J. Mater. Sci. 111574

[4] Nelson J K 1993 Breakdown strength of solids Engineering Dielectrics vol 2A (Philadelphia, PA: ASTM)

[5] Crine J-P and Vijh A K 1985 Molecular approach to the physico-chemical factors in the electrical breakdown of polymers Appl. Phys. Commun. 5 139-63

[6] Dissado L A and Fothergill J C 1992 Electrical Degradation and Breakdown in Polymers (London: Peter Peregrinus)

[7] Nelson J K, Fothergill J C, Dissado L A and Peasgood W 2002 Towards an understanding of nanometric dielectrics Annual Report of the Conf. on Electrical Insulation and Dielectric Phenomena (Piscataway, NJ: IEEE) pp 295-8

[8] Jonscher A K 1983 Dielectric Relaxation In Solids (London: Chelsea Dielectrics Press)

[9] Dissado L A and Hill R M 1984 Anomalous low frequency dispersion. A near DC conductivity in disordered low dimensional materials J. Chem. Soc. Faraday Trans. II 80 291-319

[10] Chapman D L 1913 A contribution to the theory of electrocapillarity Phil. Mag. 25 475-81

[11] Tschöpe A 2001 Grain size-dependent electrical conductivity of polycrystalline cerium oxide II: space charge model Solid State Ion. $139267-80$

[12] Lewis T J 2003 private communication

[13] Alison J 1998 A high field pulsed electro-acoustic apparatus for space charge and external circuit current measurement within solid dielectrics Meas. Sci. Technol. 9 1737-50

[14] Bambery K R and Fleming R J 2003 Activation energies and electron transport in LDPE Annual Report on IEEE Conf. on Electrical Insulation and Dielectric Phenomena pub no. 03CH37471 (Piscataway, NJ: IEEE) pp 28-31

[15] Griseri V 2000 The effects of high electric fields on an epoxy resin PhD Thesis University of Leicester, UK

[16] Mason J H 1955 Breakdown of solids in divergent fields Proc. IEE 102C 254-63 
[17] Griseri V et al 2002 Electroluminescence excitation mechanisms in an epoxy resin under divergent and uniform field Trans. IEEE 9 150-60

[18] Kawamoto A et al 1991 Effects of interface on electrical conduction in epoxy resin composites Proc. 3rd Int. Conf. on Properties and Applications of Dielectric Materials (Piscataway, NJ: IEEE) pp 619-22

[19] Schmidt-Rohr K and Spiess H W 1991 Nature of non-exponential loss of correlation above the glass transition investigated by multidimensional NMR Phys. Rev. Lett. 663020

[20] Henk P O, Kortsen T W and Kvarts T 1999 Increasing the electrical discharge endurance of acid anhydride cured
DGEBA epoxy resin by dispersion of nanoparticle silica High Perform. Polym. 11 281-96

[21] Henk P O, Henriksen M, Holbell J T and Iqbal J 1992 The influence of various mineral fillers in epoxy plastics on the resistance to partial discharges Proc. Nordic Insulation Symp. (Trondheim) pp 4.2:1-7

[22] Artbauer J 1996 The electric strength of polymers J. Phys. D: Appl. Phys. 29 446-56

[23] van Turnhout J 1980 Electrets (Springer Topics in Applied Physics vol 33) ed G M Seessler (Berlin: Springer) chapter 3

[24] Sternstein S S and Zhu A-J 2002 Reinforcement mechanism of nanofilled polymer melts as elucidated by nonlinear viscoelastic behaviour Macromolecules 35 7262-73 